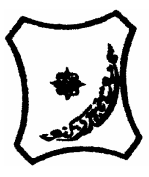

Bayero Journal of Pure and Applied Sciences, 1(1):25 - 28

Received: August, 2008

Accepted: November, 2008

\title{
THE GENETIC AND MOLECULAR BASIS OF BACTERIAL INVASION OF EPITHELIAL CELLS - A REVIEW
}

\author{
${ }^{1}$ Usman, A. D.* ${ }^{1}$. Arzai, A. H. , and ${ }^{2}$ Sulaiman, S. K. \\ ${ }^{1}$ Department of Biological Sciences, Bayero University, Kano. P.M.B. 3310, Kano. \\ ${ }^{2}$ Biology Department, Katsina state University, Katsina. \\ * Correspondence Author
}

ABSTRACT

In this review, two of the most important virulence factors of bacterial invasion of epithelial cells (adhesion and invasion) were studied from the genetic and molecular point of view. Other virulence factors are; attachment to host cells multiplication within the host, interference with host defense systems, and damage to the host. These are the determinants of microbial pathogenicity. Animal cells in tissue cultures (in-vitro) were used to study adherence and invasive properties of pathogenic bacteria. Invasion of epithelial cells was demonstrated to be triggered by invasion plasmid antigens B, C, and D (IpaB, IpaC and IpaD) which is accomplished by intracellular spread gene icsA. The invasion of epithelial cells by some individual species of bacteria were also reviewed.Yersinia enterocolitica invasiveness was shown to be mediated by inv gene product, invasin. Salmonella typhi was found to have four invasion loci; inv A-D, but $H$ genes are contained in 33kb DNA fragment. Enteropathogenic E. coli (EPEC) achieve invasion by assembly of highly organized cytoskeletal structures in the epithelial cells just beneath the adherent bacteria, while Shigella flexneri was found to harbor large plasmid which encodes or regulates some functions required for epithelial cells penetration. Generally, virulence factors in bacteria may be encoded on chromosomal DNA, bacteriophage DNA, and plasmids or transposons.

Key words: Genetics, Molecular, Epithelial cells, Invasion, Pathogenicity and Virulence

\section{INTRODUCTION}

The capacity of bacteria to invade, become established and multiply in tissues is a very important aspect of bacterial pathogenicity. If the damages is severe, the host will die, in general this is detrimental to the bacteria as well since they destroy the source of their livelihood.

The pathogenic species of bacteria are of great medical importance as causative agents of infectious diseases. Moreover, as the condition of human existence have changed, so have the bacterial species that produce diseases. It is against this background that molecular genetics have now entered the field of microbial pathogenicity. The concepts and methodologies that have been developed in the area of gene structure and gene expression are now being applied to the genetic basis of invasiveness.

It is the hope of any review of this nature that understanding of mechanisms of pathogenicity at the molecular level will lead to new control measures for infectious diseases and that insight gained through understanding of the genetic mechanisms will enable us to cope more effectively with pathogenic bacteria.

Animal cells in tissue cultures have frequently been used in in-vitro assays to examine the adherence and invasive properties of pathogenic bacteria (Lee et al., 1977). Attachment of some enteropathogenic bacteria to epithelial cells takes place through specific adhesion (Ligand) receptors
(Fletcher, 1980). For example some strains of enterotoxigenic $E$. coli bear mannose resistant haemagglutinating fimbrial adhesin (Evans et al., 1978) that are specific for receptors on the epithelial cells (Lindah et al., 1981). Fimbrae are now however responsible for epithelial cells attachment by all enteropathogenic bacteria (Deneke et al., 1985).

There is evidence that epithelial cell association results from non-specific physicochemical interaction such as surface change and hydrophobicity and that fimbrae only promote epithelial cell association by overcoming electrical repulsion forces and increasing hydrophobicity (Schiemann, et al., 1987).

According to the studies of Schiemann, et a.I, (1987), interaction of bacteria with epithelial cell in-vitro places bacteria into four possible locations with respect to cells:- i) free and unassociated, ii) attached to in animate surfaces, iii) attached to the epithelial cell surface and, iv) inside the epithelial cells.

Entry into the host (Epithelial cells invasion) Entry involves either direct invasion of the epithelial cells of a mucus membrane or passage between them followed by invasion of the deeper tissues. Not all pathogens invade; Vibrio cholerae causes disease by toxin production from within the intestinal lumen. 
Invasion of epithelial cells by the gut pathogen Shigella flexneri has been investigated in some detailed studies by Sansonetti (1992). Entry of Shigella into cells is triggered by three gene products IpaB (invasion plasmid antigen B), IpaC and IpaD. IpaB is a haemolysin responsible for the release of Shigella from the vacuole into the cytoplasm where the organisms then move by polymerisation of host actin under the influence of the intracellular spread gene ics A (Sansonetti, 1992). This motility results in the generation of protrusions from one cell into the next. The protrusion according to Mitchell (2007) is clipped off to form a vacuole within the next cell. The double membrane of this vacuole are then lysed by the product of the icsB gene, the organisms are released into the cytoplasm and the cycle begins (Mitchell, 2007).

\section{The Genetic and Molecular Mechanism of Bacterial Epithelial Cells Invasion}

In an attempt to study the genetics of invasiveness, Formal and Hornick (1978), obtained hybrids from mating of non virulent $\mathrm{E}$. coli strains with Shigella flexneri strains. They were tested for invasiveness in experimental animals. It was found that replacement of certain segments of Shigella chromosomes by the corresponding segment of E. coli chromosome resulted in the loss of virulence while replacement of other segment did not. It was concluded that pathogenicity is determined by a multiplicity of genes.

Virulence factors in bacteria may be encoded on chromosomal DNA, bacteriophage DNA, plasmids, or transposons, in either plasmid or bacteria1 chromosome (Johnny, 2007). For example, the capacity of the Shigella species to invade cells is a property encoded in part on a 140 mega-dalton plasmid. Similarly, the heat-Labile enterotoxin (LT I) of $E$. coli is plasmid encoded, whereas the heat labile toxin (LT II) is encoded on the chromosome. Other virulence factors are acquired by bacteria following infection by a particular bacteriophage, which integrates its genome into the bacterial chromosome by the process of lysogeny (Johnny, 2007).

In general, not much work has been done on the genetics of invasiveness in other bacteria than Enterobacteriaceae especially Yersinia, Enteropathogenic E. coli (EPEC), Salmonella, Shigella, and Listeria monocytogenes.

\section{(i) Yersinia invasiveness}

The best characterized invasion process is mediated by the Yersinia inv gene product, invasin. The $Y$ enterocolitica and $Y$ pseudotuberculosis inv genes are highly homologous and encode invasion at 92 or 103 KD outer membrane protein (Young et al, 1990). Invasin promotes invasion by interacting with eukaryotic transmembrane proteins belonging to the integrin superfamily (Isberge and Leong, 1990). These integrins are often localized in focal contacts, participate in transducing extracellular signals, and are components of the apparatus that anchors the cell cytoskeleton to the membrane and extracellular surfaces (Hynes, 1992).

\section{(ii) Salmonella invasiveness}

Adherence of Salmonella typhimurium to the epithelial cell surface occurs only with viable, metabolically active bacteria (Finlay et al., 1989). Salmonella typhimurium invasion is also associated with transient increase of inositol phosphate concentration in the host cytoplasm (Ruschkowski et al., 1992). In $S$. typhi, four invasion loci, distinct from $S$. typhimurium inv $A-D, H$ genes are contained in a $33 k b$ DNA fragment (Elsinghorst et al., 1989). This fragment, but not the equivalent region from Salmonella typhimurium, confers to transform $E$. coli low levels of invasiveness. Elsinghorst et al., (1989) have cloned a chromosomal region that confers Escherichia coli HB101 the ability to invade cultured human intestinal epithelial cells from $S$. typhi Ty2. At the end of their experiment three invasion-positive recombinant cosmids were isolated and restriction endonuclease analyses showed a 33-kilobase region of identity. Transmission electron microscopy of the epithelial cells invaded by $S$. typhi Ty2 or. E. coli $\mathrm{BH} 101$ carrying an invasion cosmid showed intracellular bacteria contained within endocytic vacuoles.

\section{(iii) Entropathogenic Escherichia coli (EPEC) invasiveness}

When EPEC adhere to the epithelial cells surface, they elicit an attaching and effacing phenotype which involves intimate contact between the bacteria and infected cells resulting in localized degeneration of the brush border microvilli (Moon et al., 1983). Another manifestation of this phenotype according to Knutton et al. (1989), is the assembly of highly organized cytoskeletal structures in the epithelial cell just beneath the adherent bacteria. This structure is composed of cytoskletal elements such as actin filaments, alpha-actin, talin, ezrin, and tyrosin phosphorylated proteins (Knutton et al., 1989). Mutants deficient in the ability to elicit cytoskeletal rearrangement or attaching and effacing phenotype are non- invasive.

\section{(iv) Shigella invasiveness}

Shigella flexneri strains were studied to determine whether plasmids are involved in their virulence. All invasive $S$. flexneri strains, irrespective of serotype, were found to harbour a large plasmid (Sansonetti et al., 1982). It was evidently concluded therefore, that this large $S$. flexneri plasmid encodes or regulates some functions required for epithelial cell penetration (Sansonetti et al., 1982). 


\section{DISCUSSION}

Molecular genetics is beginning to throw more light on the mechanisms of bacterial epithelial cell invasion. It helps in revealing that the genes controlling virulence are often located on extrachoromosomal elements, which facilitate their cloning, sequencing and gene expression.

Molecular genetics, through studies of bacterial invasion of epithelial cells, has provided a means of attacking practical problems. For example, it has been suggested that the virulence plasmid in Yersinia enterocolitica controls epithelial cell attachment but not invasion. Portnoy et al. (1980) in this regard concluded that invasion of HEP-2 cells was not plasmid mediated. Schiemann et al. (1987) and Vesikari (1981), reported that plasmid positive $Y$. enterocolitica secotypes $0: 3$ and $0: 9$ grown at $37^{\circ} \mathrm{C}$ were adherent but not invasive for HEP-2 cells. Whereas plasmid-negative cultures were invasive but not adherent.

Generally, Enteropathogenic bacteria have been demonstrated to exhibit physicochemical surface properties that promote adhesion; which involves unique or specific bonding mechanism. It could be derived also that the protein invasin, promoted both attachment and invasion of cultured epithelial cells; the two functions which are not generally separable by insertional mutagenesis.

\section{REFERENCES}

Deneke, C.F., McGowan, K. and Gorbach, S.L. (1985) Sulfhydryl modifying agents prevent the attachments of enteropathogenic E. coli (ETEC) to human small intestinal cells. Journal of medical microbiology 20:205 218

Evans, D.G., Evans D.J., Tjawo, S. and Dupont, H.L. (1978). Detection and characterization of colonization factor of enterotoxigenic $\mathrm{E}$. coli isolated from adults with diarrrhoea. Infection and Immunity 19:727 - 736.

Elsinghorst, E.A., Baron, L.S. and Kopecko, D.J. (1989). Penetration of human intestinal and epithelial cells by Salmonella. Molecular cloning and expression of $\mathrm{S}$. tuphi invasion determinants in E. coli. Proc. Nattl. Acad. Sci. USA 86:51737. In Bioassay Vol. 15, No.1, Jan. 1993.

Finlay, B.B., Hefferon, F. and Falkow, S. (1989). Epithelial cell surface induce Salmonella protein required for bacterial adherence and invasion Science 243:940-3.

Finlay, B.B and Falkow, S. (1989) Common theme in microbial pathogenicity. Microibol.. Rev. 53:210-230

Fletcher, M. (1980). The question of passive versus active attachment mechanisms in nonspecific bacterial adhesion. In Berkeley R.C.W. et al (eds) Microbial adhesion to surfaces, Ellis Herwood Ltd Chichester, pp 197-210.
It was also demonstrated, in the studies of the molecular mechanism of bacterial attachment to host cells by Finlay and Falcon, (1989), that bacteria associated with diarrhoeal disease in humans possess colonization factor antigens I and II (CFA/I and CFA/II), which at the same time function as adhesins. The genes encoding CFA pili are probably always plasmid-borne, and strains that loose this plasmid also lose both virulence and ability to adhere to intestinal mucous membrane. Further evidence that these pili play an essential role in pathogenesis comes from the observation that animals immunized against purified CFA/I and CFA/II are protected from strains that bear these pili.

\section{CONCLUSION}

The ability of bacteria to resist host defenses by virulence factors has extensively been studied, but only few mechanisms exhibited by most pathogenic bacteria were understood by molecular geneticist, while much remain obscure. However, at molecular level, it has been demonstrated that polysaccharide capsules, proteins toxins, lipopolysaccharides help bacteria to over come host defense system. It is hoped that further research and development in the field of molecular genetics will shed more light on the processes underlying resistance to host defense.

Formal, S.B., and Hornick, R.B. (1978). Invasive E. coli. Journal of Infectious Diseases. 137:6414

Hynes, R.O. (1992). Integrins: versatility, modulations and signalling in cell adhesion. Cel/ 69:11-25

Iseberge, R.R. and Leong, J.M. (1990). Multiple Bet-1 chain integrins are receptors for invasin, a protein that promotes bacterial penetration into mammalian cells. Cell, 60: 861-71.

Johnny, W.P. (2007). Bacterial pathogenesis. In: http: //www.gsbs.utmb.edu/microbook/ch 007. htm.

Knutton, S. Baldwin, T., Williams, P.H. and McnNeish, A. (1989): Actin accumulation at sites of bacterial adhesion to tissue culture cells: basis of a new diagonostic test for enteorpathogenic $E$. coli. Infection and Immunity, 57:1290-8.

Lee, W.H., Mcgrath, P.P., Carter, P.H. and Eide, E.L (1977). The ability of some Yersinia enterocolitica strains to invade Hela cells. Canadian Journal of microbiology 23:171422.

Lindahl,M., Faris, A., Wadstron, T. and Hjerten, S. (1981): A new test based on "salting out" to measure relative surface hydrophobicity of bacterial cells. Biochem. Biophys. Acta. 677: 471 - 476, In: J. Med. Microbiol. 28:205-218.

Mitchell, T.J. (2007). Molecular basis of Virulence In http://adc. Bmj.com/cgi/content/full/78/3/197. 
Moon, H.W., Whipp, S.C., Argenzio, R.A., Levine M.M. and Gianella, R.A. (1983). Attaching and Effacing activities of rabbit and human enteropathogenic $E$. coli in pig and rabbit intestine. Infection and Immunity, 41:1340 51.

Portnoy, D.A., Moseley, S.L. and Falkow, S. (1980). Possible plasmid mediated determinates of pathogenesis in Y. entercolitica. In: Abstracts of the annual meeting of the American Society of Microbiology pp. 19 Washington, D.C. American Society of Microbiology.

Ruschkowski, S., Rosenshine, I. and Finlay, B.B. (1992) Salmonella typhimurium induce an inositol phosphate flux in infected epithelial cells. FEMS letters 95: 121-126: In: Bioassays Vol. 15 No.1 Jan. 1993.

Sansonetti, P.J. (1992). Molecular and cellular biology of invasion by Shigella flexneri and other enteroinvasive pathogens. Symposia of the Society for General Microbiology 49:47-60
Sansonetti; P.J., Schiemann, D.A. and Devenish, J.A. (1982). Relationship of Hela cell infectivity to biochemical, serological and Virulence characteristic of $Y$. enterocolitica. Infection and Immunity 35:497-506.

Schiemann, D.A., Marlys, R. Crane, P.J. and Swan, Z. (1987). Attachment of Yersinia entrlocolitica to epithelial cells. Journal of Medical Microbiology 28:205-218

Vesikari, T. (1981). Plasmids in $Y$. enterocolitica serotypes $0: 3$ and 0:9 correlation with epithelial cell adherence in.-vitro. Infection and Immunity 33:870-876.

Young V.B., Miller, V.L., Falkow, S. and Schoolink, G.K. (1990). Sequence localization and functions of the invasin protein of $Y$. enterolocitica. Molecular Microbiology. 4(7): $1119-1128$. 